Metal-organic extended 2D structures: Fe-PTCDA on Au(111)

This article has been downloaded from IOPscience. Please scroll down to see the full text article.

2010 Nanotechnology 21305703

(http://iopscience.iop.org/0957-4484/21/30/305703)

View the table of contents for this issue, or go to the journal homepage for more

Download details:

IP Address: 150.244.101.30

The article was downloaded on 09/07/2010 at 10:28

Please note that terms and conditions apply. 


\title{
Metal-organic extended 2D structures: Fe-PTCDA on Au(111)
}

\author{
Lucía Álvarez ${ }^{1}$, Samuel Peláez ${ }^{2}$, Renaud Caillard ${ }^{1}$, \\ Pedro A Serena ${ }^{2}$, José A Martín-Gago ${ }^{1}$ and Javier Méndez ${ }^{1}$ \\ ${ }^{1}$ Grupo ESISNA, Instituto de Ciencia de Materiales de Madrid, Consejo Superior de \\ Investigaciones Científicas (CSIC), c/ Sor Juana Inés de la Cruz 3, Campus de Cantoblanco, \\ E-28049 Madrid, Spain \\ ${ }^{2}$ Grupo de Teoría y Simulación de Materiales, Instituto de Ciencia de Materiales de Madrid, \\ Consejo Superior de Investigaciones Científicas (CSIC), c/ Sor Juana Inés de la Cruz 3, \\ Campus de Cantoblanco, E-28049 Madrid, Spain \\ E-mail: jmendez@icmm.csic.es
}

Received 23 March 2010, in final form 31 May 2010

Published 6 July 2010

Online at stacks.iop.org/Nano/21/305703

\begin{abstract}
In this work we combine organic molecules of 3,4,9,10-perylenetetracarboxylic dianhydride (PTCDA) with iron atoms on an Au (111) substrate in ultra-high vacuum conditions at different temperatures. By means of scanning tunnelling microscopy (STM) we study the formation of stable 2D metal-organic structures. We show that at certain growth conditions (temperature, time and coverage) stable 'ladder-like' nanostructures are obtained. These are the result of connecting together two metal-organic chains through PTCDA molecules placed perpendicularly, as rungs of a ladder. These structures, stable up to $450 \mathrm{~K}$, can be extended in a 2D layer covering the entire surface and presenting different rotation domains. STM images at both polarities show a contrast reversal between the two molecules at the unit cell. By means of density functional theory (DFT) calculations, we confirm the stability of these structures and that their molecular orbitals are placed separately at the different molecules.
\end{abstract}

(Some figures in this article are in colour only in the electronic version)

\section{Introduction}

Supramolecular assembly of complexes [1] has become a relevant issue in the scientific community [2, 3] since several investigations have successfully shown the potential applications of molecular assemblies in the fields of magnetism, optical applications and catalysis [4-7]. A possible strategy to obtain these assemblies is to combine different organic molecules leading to ordered domains with different stoichiometries [8-10]. Other efforts focus on the combination of organic molecules with metals, in order to form metalorganic complexes [11]. In most cases these complexes are formed by a dehydrogenation process [12-15]. In this work we will address the study on the formation conditions and stability of extended 2D molecular layers covering large surface regions. The molecular species we have used is 3,4,9,10-perylene-tetracarboxylic-dianhydride (PTCDA), a planar molecule well known in the literature as an archetypal organic semiconducting molecule used for growth studies of organic molecules on various substrates [16-18]. PTCDA molecules grow on the $\mathrm{Au}(111)$ substrate forming wellordered monolayers with a 'herring-bone' structure [19] that is stabilized through hydrogen bonds between the molecules. The PTCDA molecular layer exhibits a weak substrate interaction [20] with molecules separated from the substrate by about $0.33 \mathrm{~nm}[21,22]$. Previously, we have reported the formation of stable coordination compounds made of PTCDA molecules coordinated with iron atoms to form higher order molecular structures as nanodots or metal-organic chains $[23,24]$. In this paper we investigate the experimental conditions to form ordered 2D metal-organic structures based on the combination of PTCDA molecules and iron on a $\mathrm{Au}(111)$ surface. Also, we observe changes in the contrast of the STM images when measuring them at both bias polarities. By density functional theory (DFT) calculations we confirm the stability of these 2D structures, and we correlate the changes in the contrast with the charge transfer processes between the molecules forming the structure. 


\section{Experimental details}

Experiments were performed in an ultra-high vacuum (UHV) chamber with a base pressure in the range of $4 \times 10^{-10}$ mbar. The chamber is also equipped with a quadrupole mass spectrometer (QMS), Auger electron spectroscopy (AES) and low energy electron diffraction (LEED). A gold single crystal with (111) orientation was used as substrate. The Au(111) surface was prepared by repeated cycles of $\mathrm{Ar}^{+}$sputtering and subsequent annealing. This process is repeated until a typical LEED-pattern is obtained and no signal of carbon or another impurity is observed by AES. STM analysis of the substrate so prepared reveals wide clean terraces $(\sim 200 \mathrm{~nm}$ wide) with the characteristic 'herring-bone' reconstruction and no significant traces of contaminants. Iron was evaporated from a homebuilt evaporator by electron bombardment of an iron rod. PTCDA purified molecules were introduced into the chamber in a crucible and evaporated via sublimation at $530 \mathrm{~K}$. Both evaporators were degassed (PTCDA at $510 \mathrm{~K}$ ) for hours before deposition and they were used at very low rates, below $0.1 \AA \mathrm{min}^{-1}$, so the pressure is kept in the $10^{-9} \mathrm{mbar}$ range during evaporation. Iron and PTCDA were deposited on the gold substrate following different deposition schemes: both simultaneously or one after the other. After deposition of both materials, the samples were annealed up to 360-390 K for $10 \mathrm{~min}$ to achieve the formation of well-ordered structures. Ultra-sharp STM tips were made of tungsten electrochemically etched in a solution of $\mathrm{KOH}$. Then they were cleaned and sharpened in UHV by annealing and high voltage until stable field emission was obtained [25].

Tunnelling measurements were performed using a commercial variable temperature STM setup from Omicron (VTSTM) in a temperature range of 100-400 K. The microscope was operated using Nanotec's electronics software [26], mainly in constant current mode.

\section{Computational details}

In order to verify the stability of the proposed molecular models, we have performed theoretical calculations using the DMol3 software [27, 28]. This software allows an optimization of the molecular structures based on DFT. The exchange-correlation energy term has been described within the local density approximation (LDA) [29]. All the electrons are described by sets of localized numerical atomic orbitals, thus pseudopotentials are not required. Geometry optimization of the molecular models was done by standard conjugate gradients (CG) minimization of the total energy. Final convergence was assumed when the energy change in a CG step was below $1 \times 10^{-5}$ Hartree and either the energy gradient or the maximum atomic displacement were below their threshold values $\left(0.002\right.$ Hartree $\AA^{-1}$ and $0.005 \AA$, respectively). After convergence the optimized structure is fully characterized by its geometry, the electronic spectrum (in particular the highest occupied molecular orbital (HOMO) and the lowest unoccupied molecular orbital (LUMO)) and the charge distribution.

\section{Results and discussion}

\subsection{Structural results}

It is well known that deposition of iron atoms on a $\mathrm{Au}(111)$ substrate leads to the formation of iron islands pinned at the corners of the 'herring-bone' reconstruction [30]. Moreover, subsequent deposition of PTCDA molecules on the Fe-patterned surface produces nucleation of the organic molecules around the iron islands [23]. We have previously reported that annealing the resulting PTCDA-Fe-Au(111) surface up to $370 \mathrm{~K}$ promotes the formation of elongated 1D nanostructures or chains [23, 24]. Increasing the PTCDA coverage and annealing up to $360-390 \mathrm{~K}$ produces a new morphology consisting of parallel chains linked by extra PTCDA molecules. This 'ladder-like' structure, as shown in figure 1(a), is formed by two chains joined together through perpendicular PTCDA molecules, placed as the rungs of a ladder. The proposed molecular model is shown in figure 1(b). Notice that this configuration is formed by two kinds of nonequivalent PTCDA molecules since molecules forming the long 1D chain interact with four iron atoms, while those molecules at rung positions interact only with two iron atoms.

By increasing proportionally PTCDA and iron coverages, the 'ladder-like' structure can be progressively extended to form $2 \mathrm{D}$ structures covering the gold surface. The degree of order can be improved by co-evaporation and specially by further annealing up to 360-390 K. At room temperature most of the iron is pinned at the elbows of the reconstruction [30]. However, through annealing the iron diffusion is enhanced and the PTCDA molecules and iron atoms are more likely to encounter each other and interact forming the metalorganic structures. In figure 2 we present an STM image of the substrate with a coverage close to $0.3 \mathrm{ML}^{3}$ which provides a hint of the growing from chains to the 'ladderlike' nanostructures at room temperature. Nucleation at the lower part of the steps is also observed. We notice that there are no clear preferential directions for the obtained structures similarly to the single metal-organic chains. Nevertheless, in some images the direction [11ㄹ] , which connects the gold reconstruction elbows, appears visible as the prevailing direction. These 2D arrangements present typically a width that is a multiple of the separation between the two parallel chains forming the ladder-like structure proposed in figure 1(b). In figure 2 we can observe widths corresponding to one, two and three parallel ladders. The molecules at 'rung' positions are not stable without the presence of two 'chain' structures. Some images show blurry lines at the sides of the structure, corresponding to PTCDA molecules placed perpendicularly (rungs molecules).

The process of formation of metal-organic structures is controlled by the encounter on the surface between both components: PTCDA and iron. Since PTCDA molecules are weakly interacting with the gold [20,21], and therefore highly diffusing over the substrate, the process is controlled by the slower iron diffusion. Iron atoms can be pinned

\footnotetext{
3 Measured coverages of the metal-organic structures does not correspond with the nominal ones obtained by separated evaporation of Fe and PTCDA
} on clean gold due to a decrease of the sticking coefficients. 

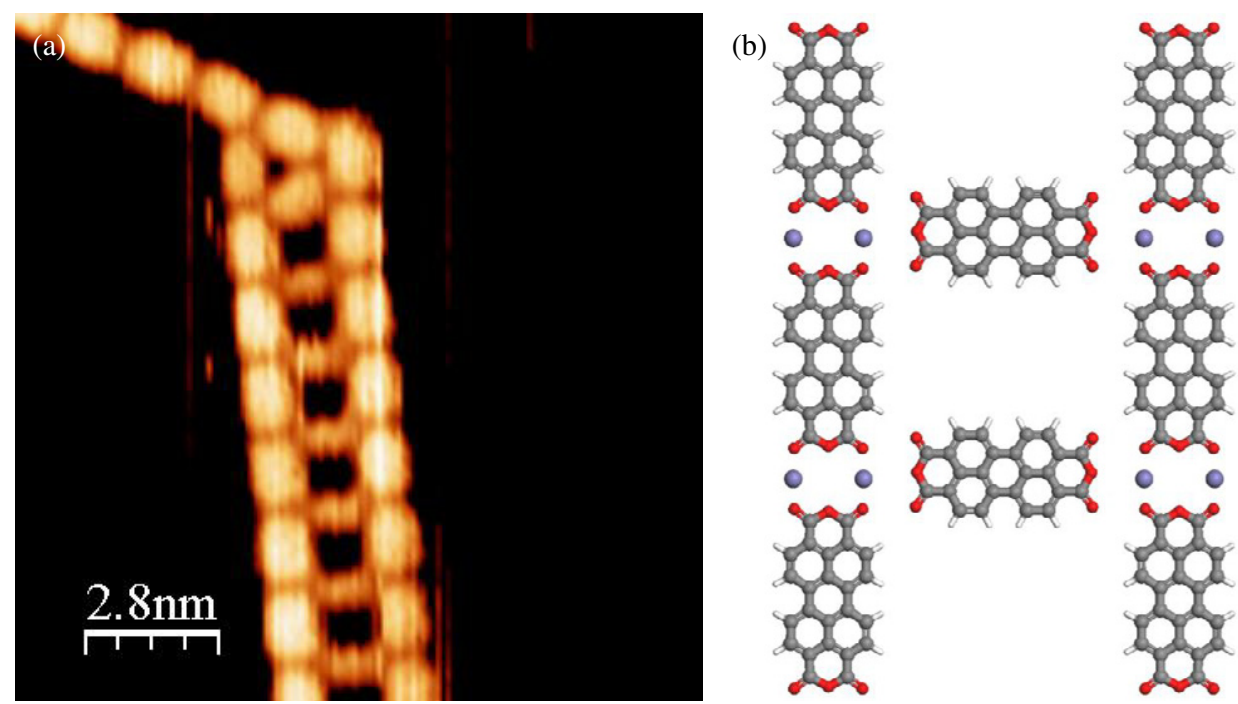

Figure 1. (a) STM image of the 'ladder-like' nanostructure $(14 \mathrm{~nm} \times 14 \mathrm{~nm}) . V=-1.3 \mathrm{~V} ; I=0.47 \mathrm{nA}$. (b) Model of the 'ladder-like' structure: two Fe atoms link consecutive PTCDA molecules forming the chain and perpendicular PTCDA molecules join the chains. Hydrogen atoms in white, oxygen in red, carbon in grey and iron in violet.

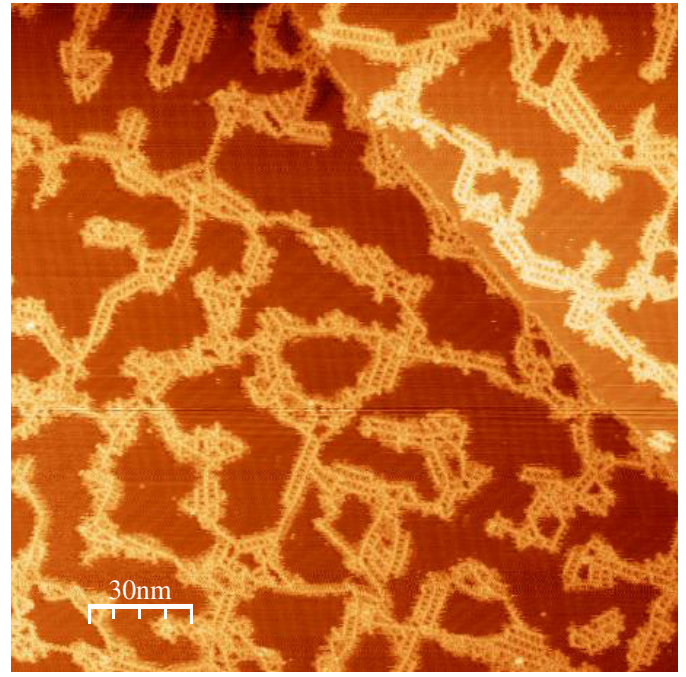

Figure 2. Formation of connected metal-organic structures. STM image $(150 \mathrm{~nm} \times 150 \mathrm{~nm})$ measured at RT for a coverage close to $0.3 \mathrm{ML}$ where the structures start to connect between them. $V=-1 \mathrm{~V} ; I=0.5 \mathrm{nA}$.

at reconstruction elbows or steps. Thus, when the iron evaporation is made first, the formation of metal-organic structures is localized at steps or at reconstruction elbows. By co-evaporation (simultaneous evaporation of PTCDA and iron) or by further annealing, the diffusion of iron atoms is assured, and metal-organic structures are formed all over the surface.

The effect of the annealing can be observed in figure 3 . In this image the substrate has been covered with about one monolayer of PTCDA (0.9 ML) and the corresponding iron $(0.07 \mathrm{ML})$ (see footnote 3 ). The sample was then annealed up to $390 \mathrm{~K}$. As a result we observe the formation of ordered domains with the 'extended ladder-like' structure. The image of figure 3 shows that the extended structure covers almost completely the surface in a 2D metal-organic structure. PTCDA patches (at the left side of the image) and areas with bare gold (at the lower part and in the centre of the image) can be also observed. The existence of pure PTCDA regions is due to an excess of PTCDA with respect to the Fe. Similarly as for the PTCDA monolayer, the gold reconstruction is still visible underneath the metal-organic layer, indicating a weak interaction with the substrate. Four directional domains are visible for the extended structure, marked with letters A to $\mathrm{D}$ in the figure. Three of them, A, C and $\mathrm{D}$, form $60^{\circ}$ between them. Domain B forms an angle of $24^{\circ}$ with A. Other images (not presented) show the presence of two additional domains tilted $60^{\circ}$ with respect to the B domain. Therefore, this structure forms six domains corresponding of two nonequivalent domains with a relative angle of $24^{\circ}$ between them. For lower annealing temperatures even more domains can be observed. The presence of domains correlated with $60^{\circ}$ between them indicates the weak influence of the substrate on the formation of the structures. In figure 3(b) we present a high resolution STM image obtained at $V=-1 \mathrm{~V}$, where the intermolecular features can be viewed. The average unit cell is $1.50 \mathrm{~nm} \times 2.21 \mathrm{~nm}$. We notice that iron atoms are not imaged by STM, as also happens for other coordination structures on gold substrate [15]. A possible explanation could be a lower vertical position of the metal atoms with respect to the plane of the organic molecules.

If the annealing process is kept for a long period (several hours at $390 \mathrm{~K}$ ), we observe the formation of elongated islands with a single domain of the extended ladder-like structure (see figure 4). The long side of these rectangular islands corresponds to the chain axis of the metal-organic structure, while the shorter side is the direction of the PTCDA rung molecules. Differently from the ordered patches shown above, below these anisotropic islands the gold reconstruction appears as straight lines with no elbows. This can be noticed in the image from the white lines marked following the reconstruction directions. These reconstruction lines form an angle with the metal-organic islands close to $70^{\circ}$, or in other words, the metal-organic structure is orientated with an angle 


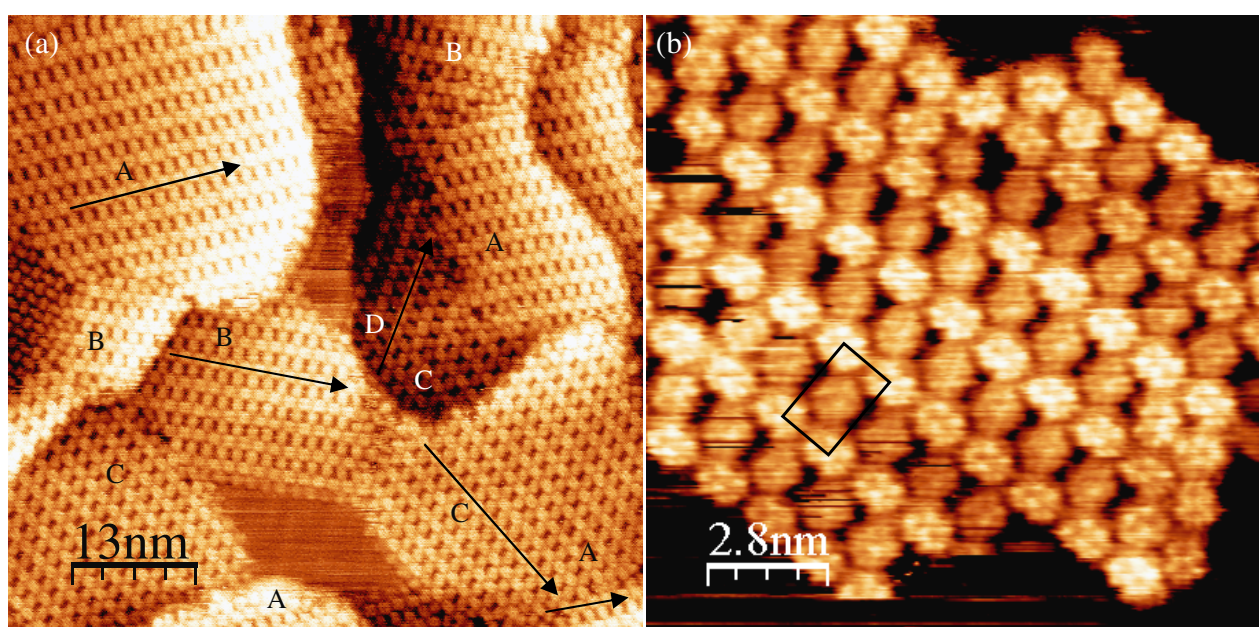

Figure 3. (a) Extended metal-organic monolayer. STM image $(65 \mathrm{~nm} \times 65 \mathrm{~nm})$ obtained at $390 \mathrm{~K} . V=-1.5 \mathrm{~V} ; I=0.23 \mathrm{nA}$. Several domains (here marked as A, B, C, D) are observed with the 'extended ladder-like' structure. (b) STM image showing a detail of the 2D extended structure $(14.2 \mathrm{~nm} \times 14.2 \mathrm{~nm}) . V=-1 \mathrm{~V} ; I=0.4 \mathrm{nA}$.

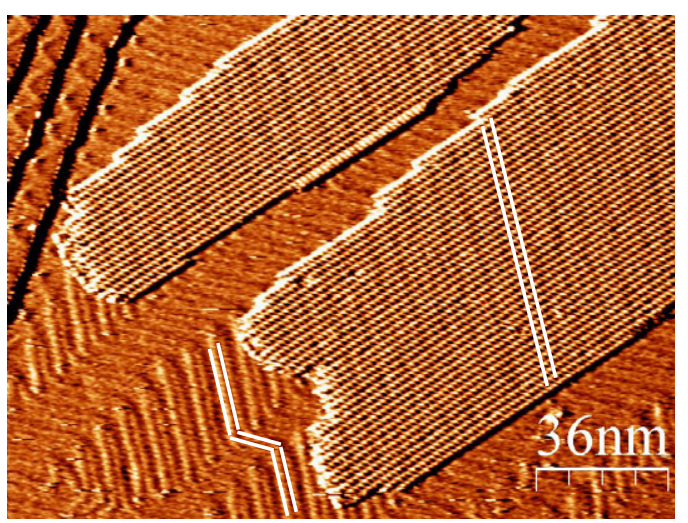

Figure 4. STM image $(180 \mathrm{~nm} \times 135 \mathrm{~nm})$ in derivative mode showing the state of the sample after a prolonged annealing. $V=-2.2 \mathrm{~V} ; I=1 \mathrm{nA}$. The metal-organic $2 \mathrm{D}$ coverage shows monodomain elongated islands. The reconstruction under the structure appears straightened with no elbows. White lines are guides for eyes showing the reconstruction lines below (straight lines) and beside (with elbows) the islands.

close to $20^{\circ}$ with respect to the [112] direction. Figure 4 shows the surface at this stage, where two islands partially cover the gold substrate. The reconstruction under them has been straightened, denoting a slight material transport of gold atoms and therefore a change of the surface stress at the gold surface.

The stability of the 2D extended structure has also been checked by annealing up to $450 \mathrm{~K}$ and by oxygen exposure ( $3 \mathrm{~L}: 1 \times 10^{-9}$ mbar for $1 \mathrm{~h}$ ). In both cases the structure remained unaltered. For the oxygen exposure inverse contrast has been observed, probably due to the adsorption of oxygen at the tip-apex.

\subsection{Spectroscopic results}

After a detailed inspection of the STM images (figures 1 and 3(b)) we notice differences between the two types of molecules forming the structure. As mentioned above, while the chain molecule is linked to four iron atoms, the rung molecule is only linked to two irons (see figure 1(b)). The chains are stable by themselves [24], denoting a stronger interaction between their molecules and molecules linking them (rungs). The difference between both kinds of molecules can be visualized by varying the bias voltage. As shown in figure 5, images at negative bias (with respect to the sample) show the chain molecules slightly brighter, while images at positive bias show the rung molecules brighter and bigger. To understand this contrast reversal we should consider a charge transfer process between the chains and the rung molecules. This charge transfer displaces the HOMO and LUMO to the chain and rungs, respectively.

In order to understand the spectroscopic data, we have carried out DFT calculations on the 'extended' ladder-like structure. For these calculations we have assumed that the interaction with the Au substrate is small enough so that it does not affect the charge distribution in the molecules. Therefore the substrate is omitted and only $\mathrm{Fe}$ atoms and PTCDA molecules are considered in the structure relaxation. We have obtained a stable structure containing two PTCDA molecules and two $\mathrm{Fe}$ atoms, as seen in figure 6. In the figure we show four unit cells of the relaxed atomic configuration obtained from a CG optimization, after 365 steps. The resulting unit cell is $2.28 \mathrm{~nm} \times 1.56 \mathrm{~nm}$ in good agreement with the experimental values $(2.21 \mathrm{~nm} \times 1.50 \mathrm{~nm})$. The top-left panel of the figure (figure 6(a)) shows the atomic configuration and the values of the electron charge for the atoms closer to the bonding region. On the lower-right panel (figure 6(d)) we show the calculated HOMO (blue-cyan) and LUMO (orange-yellow). It can be seen that the HOMO orbital is placed at the chain molecules, whereas the LUMO is on the rung molecules. This result agrees with the experimental observations at different bias shown in figure 5. Certainly, if we observe the charge distribution at the oxygen atoms (in red) shown in figure 6(a), we confirm that all the oxygen atoms in the chain molecules have more charge than those at the rungs. This is indicative of a charge transfer from the rungs to the chain molecules, mediated 

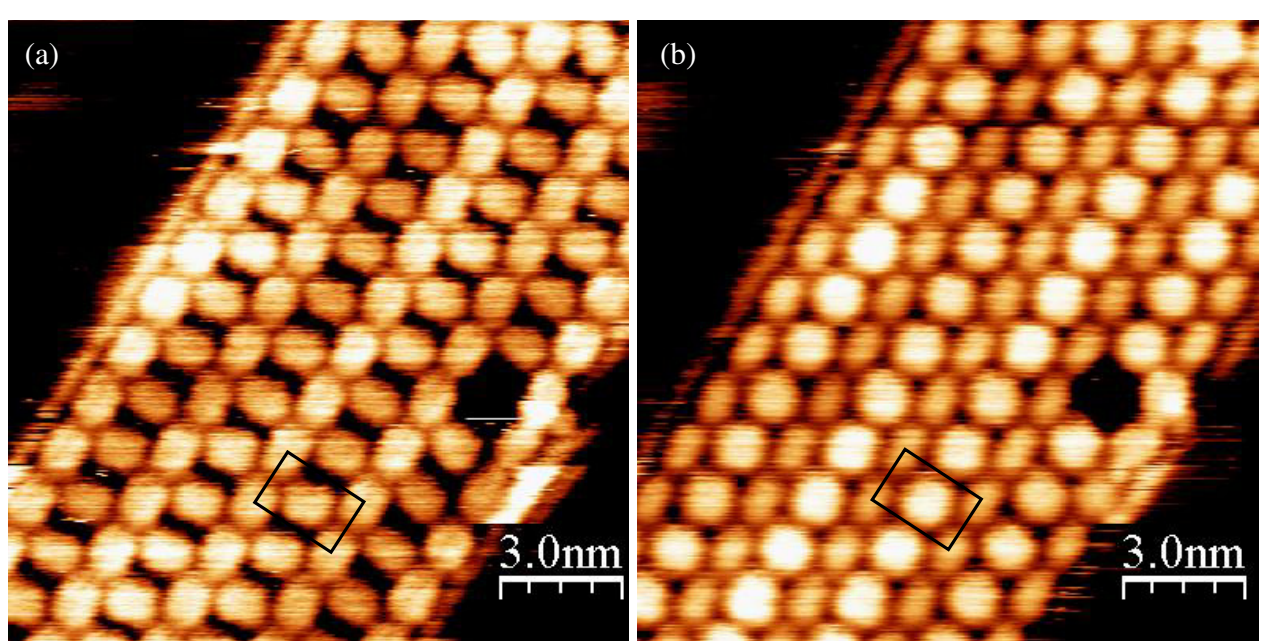

Figure 5. STM images $(15 \mathrm{~nm} \times 15 \mathrm{~nm})$ acquired at different polarities. (a) Filled-states image obtained at $V=-1.6 \mathrm{~V}$ showing the chains slightly brighter; (b) empty-states image measured at $V=+1.6 \mathrm{~V}$ showing the rung molecules brighter and bigger. The same unit cell is marked on both images.

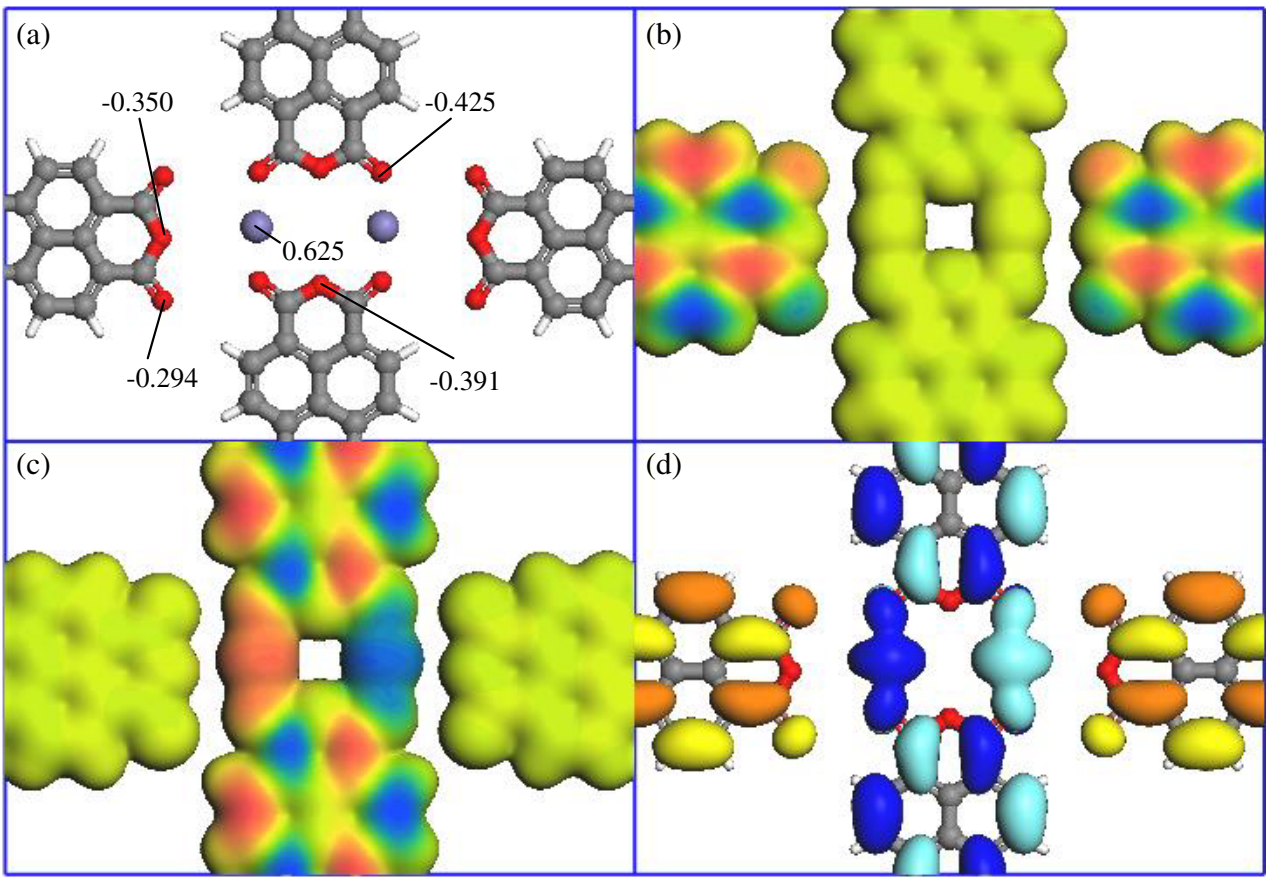

Figure 6. DFT calculation of the 2D structure. (a) Unit cell of the structure with the electron charge distribution values at the main atoms. The colour code of atoms corresponds to that of figure 1. (b) LUMO projected into the electron density. The yellow colour corresponds to the plain electron density while the red/blue colours correspond to $+/-$ deviation due to the molecular orbital. (c) HOMO projected into the electron density. (d) HOMO (blue-cyan) and LUMO (orange-yellow) displayed showing the different location of these orbitals.

by the iron atoms. The closer the atom is to the iron, the larger is the charge.

Additionally we show a representation of the LUMO and HOMO projected on the electron density. These projections shown in figures 6(b) and (c) respectively resemble closely the experimental STM images at both polarities. It should be noticed that the calculated orbitals (and the projections) of the 2D structure show internal features corresponding to the LUMO of the isolated PTCDA molecule [31, 20]. This happens for both orbitals (HOMO and LUMO) of the ladderlike structure, as shown in the panel of figure $6(\mathrm{~d})$. This fact is in agreement with intermolecular features observed experimentally. Thus, some STM images (see figure 3(b)) seem to show molecules with ten lobes, characteristic of the LUMO of the PTCDA observed at positive voltage, but here observed at negative bias voltage.

\subsection{Other structures}

Although the most stable observed structure is the ladderlike described above, we have also observed some minority areas where iron atoms and PTCDA molecules form other 

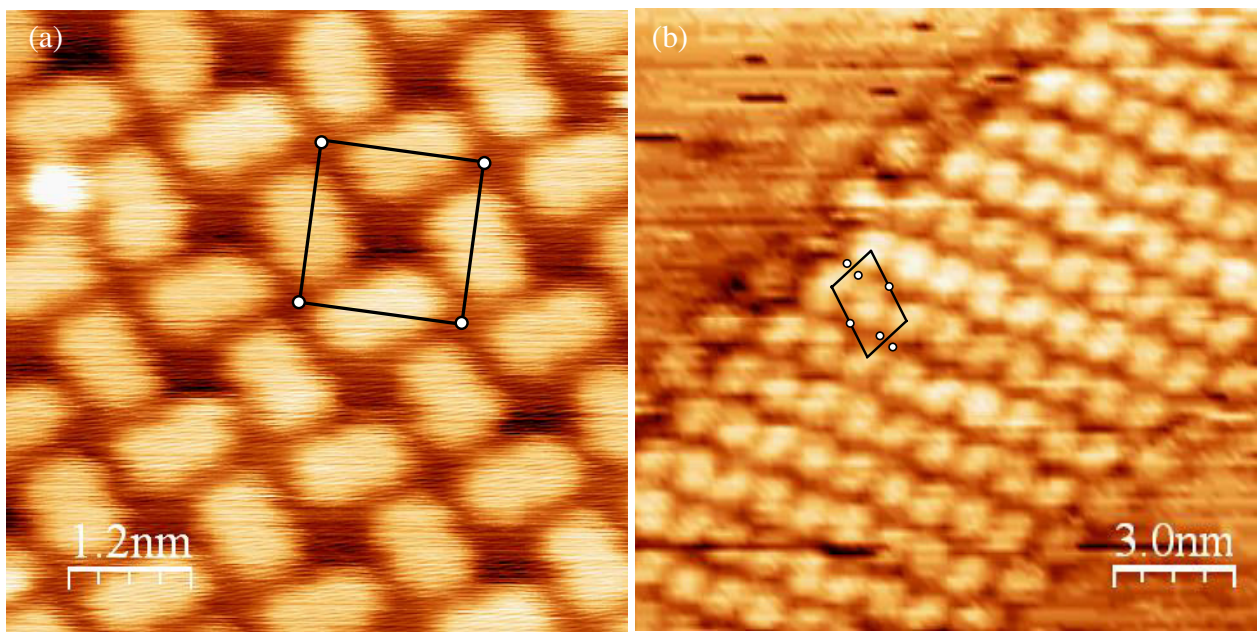

Figure 7. (a) STM image showing a tetragonal structure with a ratio of $(1: 2)$ Fe atoms per PTCDA molecules $(6 \mathrm{~nm} \times 6 \mathrm{~nm}) . V=-1.8 \mathrm{~V}$; $I=3 \mathrm{nA}$. (b) Image of an elongated metal-organic structure $(15 \mathrm{~nm} \times 15 \mathrm{~nm}) . V=+1.8 \mathrm{~V} ; I=0.5 \mathrm{nA}$.

ordered structures on gold. The origin of these structures is the local change of the Fe:PTCDA ratio. One of them is the tetragonal structure presented in figure 7(a). This structure is very similar to the square PTCDA reconstruction [19, 32], typically observed at low PTCDA coverage without iron. After a detailed inspection of the tetragonal structure (figure 7(a)), we can notice that it differs slightly from the pure PTCDA structure. This tetragonal structure corresponds to a $p 4$ symmetry (while the square structure is a $p 4 \mathrm{~mm}$ ) with two different kinds of holes between the molecules: notice the bigger separation at the centre of the marked unit cell with respect to the smaller separation at the four corners. We suggest that this hole contraction is due to the presence of one iron atom at each corner coordinating four PTCDA molecules. The resulting relation is (1:2) iron atoms per PTCDA molecule. In the absence of iron the separations are equal, as is the case of the pure PTCDA square structure. The unit cell of the square structure forms $45^{\circ}$ with the molecules, while at the tetragonal structure they are slightly tilted.

Also we have observed areas with an elongated structure forming stripes, as shown in figure 7(b). We suggest a ratio of $(3: 2)$ iron atoms per PTCDA molecule for this case. This structure can be seen as a tilted ladder-like structure. As a difference with the last, for both structures in figure 7 all the molecules are equivalent, as they are indistinguishable in images at different voltage.

\section{Conclusions}

We report the formation of stable ordered metal-organic 2D structures covering an $\mathrm{Au}(111)$ substrate. The unit cell of this ordered coverage contains two non-equivalent PTCDA molecules and two iron atoms linking them. The structure is stabilized through coordination interactions between the iron and the PTCDA molecules at the chain plus charge transfer between the chain and the perpendicular molecules. By varying the voltage bias and polarity, the image contrast between the two kinds of molecules changes and reverses.
Density functional theory calculations confirm the stability of this structure, as well as the spatial distribution of the HOMO and LUMO orbitals, which explains the contrast reversal observed in the STM images at different bias.

The 2D metal-organic layers shown in this study fall into the large family of 2D molecular networks obtained from surface self-assembly processes. There is no doubt $[2,3]$ that these $2 \mathrm{D}$ networks provide new functionality to the surfaces with several kinds of secondary templates. In this way, surface properties can be tailored for the sensing of molecular species, the confinement of molecules in order to control chemical reactions etc. The tuning and control of these $2 \mathrm{D}$ networks relies on our capacity to balance different interactions among molecules and substrate. This balance is achieved by an appropriate selection of the surface, the molecular concentration, the temperature and other external or internal parameters. Our studies reveal that temperature, PTCDA concentration and iron coverage determine the formation of several 2D nanoporous structures with different ordering, thus providing control of the kind of assembled layers grown on the surface. This is a first, but mandatory, step to control the formation of more complex structures in a controlled manner.

Regarding the possible use of our PCTDA networks to anchor different molecules we must remind readers, for the particular case we have studied, that we have found experimental and theoretical evidence of the non-uniform distribution of the electric charge between the two kinds of non-equivalent molecules forming the $2 \mathrm{D}$ network. This property is very interesting since it can be used to promote additional ordering of polar molecules adsorbed on the secondary template. In addition, we must pay attention to another interesting fact: the ordering of PCTDA molecules is associated with the formation of a 2D network of $\mathrm{Fe}$ atoms. This fact could provide interesting additional features to the whole 2D system for use in spintronics and molspintronics, one of the promising fields of application of these molecular networks. More experiments and sophisticated calculations are under way to fully understand these interesting systems. 


\section{Acknowledgments}

We acknowledge financial support from CAM projects CCG08-CSIC/MAT-3831 and CAM-S2009-MAT1467 and MICINN projects MAT2008-01497, FIS2009-13430-C01 and Consolider CSD 2007-00041. We also would like to thank J Cerdá for helpful discussions.

\section{References}

[1] Lehn J M 1995 Supramolecular Chemistry, Concepts and Perspectives (New York: VCH)

[2] Liang H, He Y, Ye Y, Xu X, Cheng F, Sun W, Shao X, Wang Y, Li J and Wu K 2009 Coordination Chem. Rev. $2532959-79$

[3] Kudernac T, Lei S, Elemans J A and De Feyter S 2009 Chem. Soc. Rev. 38 402-21

[4] Stepanow S, Lingenfelder M, Dmitriev A, Spillmann H, Delvigne E, Lin N, Deng X, Cai C, Barth J V and Kern K 2004 Nat. Mater. 3 229-33

[5] Stepanow S, Lin N and Barth J V 2008 J. Phys.: Condens. Matter 20184002

[6] Gambardella P et al 2009 Nat. Mater. 8 189-93

[7] Lee J, Farha O K, Roberts J, Scheidt Ka, Nguyen S T and Hupp J T 2009 Chem. Soc. Rev. 38 1450-9

[8] Theobald J A, Oxtoby N S, Phillips M A, Champness N R and Beton P H 2003 Nature 424 1029-31

[9] de Oteyza D G, Silanes I, Ruiz-Osés M, Barrena E, Doyle B P, Arnau A, Dosch H, Wakayama Y and Ortega J E 2009 Adv. Funct. Mater. 19 259-64

[10] Treier M, Nguyen M, Richardson N V, Pignedoli C, Passerone D and Fasel R 2009 Nano Lett. 9 126-31

[11] Barth J V 2007 Ann. Rev. Phys. Chem. 58 375-407

[12] Lin N, Dmitriev A, Weckesser J, Barth J V and Kern K 2002 Angew. Chem. Int. Edn 41 4779-83
[13] Dmitriev A, Spillmann H, Lin N, Barth J V and Kern K 2003 Angew. Chem. Int. Edn 42 2670-3

[14] Lingenfelder M A, Spillmann H, Dmitriev A, Stepanow S, Lin N, Barth J V and Kern K 2004 Chem. Eur. J. 10 1913-9

[15] Clair S, Pons S, Fabris S, Baroni S, Brune H, Kern K and Barth J V 2006 J. Phys. Chem. B 110 5627-32

[16] Forrest S R 1997 Chem. Rev. 97 1793-896

[17] Tautz F 2007 Progr. Surf. Sci. 82 479-520

[18] Umbach E, Glöckler K and Sokolowski M 1998 Surf. Sci. 402-404 20-31

[19] Mannsfeld S, Toerker M, Schmitz-Hübsch T, Sellam F, Fritz T and Leo K 2001 Org. Electron. 2 121-34

[20] Nicoara N, Román E, Gómez-Rodríguez J M, Martín-Gago J A and Méndez J 2006 Org. Electron. 7 287-94

[21] Henze S, Bauer O, Lee T, Sokolowski M and Tautz F 2007 Surf. Sci. 601 1566-73

[22] Duhm S, Gerlach A, Salzmann I, Bröker B, Johnson R, Schreiber F and Koch N 2008 Org. Electron. 9 111-8

[23] Méndez J, Caillard R, Otero G, Nicoara N and Martín-Gago J 2006 Adv. Mater. 18 2048-52

[24] Álvarez L, Caillard R, Peláez S, Serena P, Martín-Gago J A and Méndez J 2010 at press

[25] Méndez J, Gómez-Herrero J, Pascual J and Baró A M 1998 Appl. Phys. A 66 S767-9

[26] Horcas I, Fernández R, Gómez-Rodríguez J M, Colchero J, Gómez-Herrero J and Baro A M 2007 Rev. Sci. Instrum. 78013705

[27] Delley B 1990 J. Chem. Phys. 92508

[28] Delley B 2000 J. Chem. Phys. 1137756

[29] Kohn W and Sham L J 1965 Phys. Rev. A 1401133

[30] Voigtlander B, Meyer G and Amer N 1991 Surf. Sci. Lett. 255 L529-35

[31] Scholz R, Kobitski A Y, Kampen T U, Schreiber M, Zahn D R T, Jungnickel G, Elstner M, Sternberg M and Frauenheim Th 2000 Phys. Rev. B 6113659

[32] Chizhov I, Kahn A and Scoles G 2000 J. Cryst. Growth 208 449-58 